\title{
Relationships between Circulating Matrix Metalloproteinases, Tissue Inhibitor TIMP-2, and Renal Function in Patients with Myocarditis
}

\author{
Małgorzata Kobusiak-Prokopowicz ${ }^{\mathrm{a}}$ Konrad Kaaz $^{\mathrm{a}}$ Dominik Marciniak ${ }^{\mathrm{b}}$ \\ Bożena Karolko ${ }^{\mathrm{a}}$ Andrzej Mysiak ${ }^{\mathrm{a}}$ \\ aDepartment of Cardiology, Wroclaw Medical University, Wroclaw, Poland; ${ }^{\mathrm{b}}$ Department of Drugs Form Technology, \\ Wroclaw Medical University, Wroclaw, Poland
}

\section{Keywords}

Chronic kidney disease - Matrix metalloproteinases .

Myocarditis - Tissue inhibitor of metalloproteinases

\begin{abstract}
Introduction: Under physiological conditions, the myocardial extracellular matrix (ECM) is maintained by matrix metalloproteinases (MMPs) and tissue inhibitors of metalloproteinases (TIMPs). However, changes in the balance between MMPs and TIMPs can lead to pathological remodeling of the ECM, which contributes to cardiovascular and kidney diseases. The aim of our study was to assess levels of MMPs and TIMP-2 in patients with myocarditis and their relationship to renal function. Materials and Methods: Forty five patients with myocarditis who underwent CMR were included, comprising 11 with concurrent chronic kidney disease (CKD). Blood samples were obtained to assess serum levels of MMP2, MMP-3, MMP-9, and TIMP-2. Results: Serum MMP-2, MMP3 , and TIMP-2 levels negatively correlated with the ejection fraction in patients with myocarditis, while MMP-3 levels correlated with longitudinal deformation $(p<0.05)$. Serum MMP-2, MMP-3, and TIMP-2 levels also negatively correlated with renal function, as assessed by the estimated glomerular filtration rate (eGFR) $(p<0.05)$. Patients with myocarditis and
\end{abstract}

karger@karger.com www.karger.com/kbr

Karger!
(C) 2021 The Author(s)

Published by S. Karger AG, Basel

This is an Open Access article licensed under the Creative Common Attribution-NonCommercial-4.0 International License (CC BY-NC) (http://www.karger.com/Services/OpenAccessLicense), applicable to the online version of the article only. Usage and distribution for commercial purposes requires written permission. concurrent CKD had higher levels of MMP-2 and TIMP-2 than those without kidney damage. Conclusions: (1) We demonstrated that MMP-2, MMP-3, and TIMP-2 concentrations were related to left-ventricular ejection fraction, and MMP-3 levels correlated with longitudinal deformation, indicating MMPs play an important role in the post-inflammatory remodeling of the myocardium. (2) A negative correlation between the eGFR and MMP-2, MMP-3, and TIMP- 2 and a positive correlation between creatinine and MMP-3 levels indicate the role of MMPs and TIMP-2 in renal dysfunction.

(c) 2021 The Author(s).

Published by S. Karger AG, Basel

\section{Introduction}

The extracellular matrix (ECM) protects the geometry and integrity of myocardial tissues by creating a "skeleton" upon which matrix proteins can anchor, maintaining homeostasis between protein deposition and degradation $[1,2]$. ECM transformations affect various physiological processes, including cell growth and differentiation, as well as tissue development and formation [3]. The ECM is also responsible for the transmission of mechanical forces within the vessels and heart and affects the diastolic susceptibility of the arteries [4]. 
The main mediators of remodeling within the ECM are matrix metalloproteinases (MMPs), that is, enzymes with the ability to degrade structural matrix proteins. Although the production of MMPs is usually low in most tissues, certain stimuli (e.g., tissue damage, hyperglycemia, proinflammatory factors, and aldosterone) can upregulate their transcription $[5,6]$. Under physiological states, ECM remodeling by MMPs is strictly controlled by endogenous tissue inhibitors of metalloproteinases (TIMPs). However, changes in the balance between MMPs and TIMPs have been shown to be involved in pathological remodeling of the heart muscle and can contribute to acute damage during ischemia $[1,2,7]$. Indeed, there is a general tendency to increase the proteolytic activity of MMPs in the myocardium during ischemia [7]. Such irreversible ECM remodeling has been shown to be responsible for both compensatory hypertrophy and decompensated congestive heart failure [8]. Furthermore, the ventricular muscle remodeling following myocardial infarction or damage due to viral infections has also been shown to be mediated by MMPs $[8,9]$.

MMPs can be divided into 5 groups based on their substrate and structure: matrylisins, collagenases, stromelysins, gelatinases, and membrane metalloproteinases. Numbers MMP-1 to MMP-28 are used for classification. Among them, MMP-2 and MMP-9 (also called gelatinases $\mathrm{A}$ and $\mathrm{B}$ ), which digest structural proteins of the collagen-elastin network, are implicated in the pathogenesis of coronary syndromes and heart failure [10-12]. Elevated MMP-2 levels have been shown to stimulate adverse vascular remodeling and thrombotic effects, which accelerate vascular damage $[6,13]$. Increased MMP-2 and MMP-9 activity have also been shown to be associated with persistent low-grade inflammation [14] and endothelial dysfunction [15]. Therefore, MMPs may contribute to the myocardial changes observed in myocarditis.

Elevated MMP levels may also have significant effects on the kidney. Indeed, increased MMP-9 levels have been shown to be associated with kidney damage [16]. Increased MMP-3 (a stromelysin MMP) levels may also cause kidney damage, as one of the main MMP-3 substrates is type IV collagen, an important component of the basal membranes of the renal tubules and vessels [17]. MMP-2 has also been shown to increase fibrosis in the kidney [18]. Therefore, serum MMP levels may also influence kidney function in patients with myocarditis.

As serum concentrations of MMPs typically increase as TIMP levels decrease, changes in TIMP levels may also occur in cardiovascular disease. Indeed, such an imbalance in favor of proteinases is associated with increased transformation and cardiovascular disease progression [5]. There are 4 types of TIMPS (TIMP-1-4). However, different TIMPs inhibit the various MMPs better than others; for example, TIMP-2 inhibits MMP-2 more effectively than other TIMPs. Therefore, reduced levels of TIMP-2, in particular, may occur in myocarditis. The aims of the study were to: (1) assess the activity of MMPs, as well as the balance between MMPs and TIMP-2, in the serum of patients with myocarditis as elements contributing to the remodeling of the ECM and heart damage, and (2) determine the relationship between levels of MMPs and TIMP-2 and renal function.

\section{Materials and Methods}

Fifty-eight patients hospitalized in the Cardiology Department from 2013 to 2017 with myocarditis diagnosed based on the European Society of Cardiology (ESC) recommendations were qualified for the study [19]. Patients underwent standard laboratory tests, electrocardiography, and echocardiography (GE Vivid E9) during the first $24 \mathrm{~h}$ of hospitalization. Each enrolled patient had a venous blood sample drawn from an antecubital vein within 24 $\mathrm{h}$ of admission, to assess the serum levels of MMP-2, MMP-3, MMP-9, and TIMP-2. Blood samples were collected using a closed blood sampling system (BD Vacutainer ${ }^{\circledR}$ ); $4 \mathrm{~mL}$ of blood was drawn into a tube containing EDTA (for plasma recovery) and 4 $\mathrm{mL}$ into a clot activator serum tube (for serum recovery). After centrifugation and fractionation, the separated serum and plasma samples were stored at $-72^{\circ} \mathrm{C}$ until analysis. MMP-2, MMP-3, MMP-9, and TIMP-2 concentrations were determined in the preserved blood serum using R\&D Systems Quantikine ${ }^{\circledR}$ ELISA kits (Bio Techne). Within 30 days of admission, cardiac magnetic resonance imaging (MRI) was performed in patients using a General Electric Signa HDxT 1.5T scanner. For the purposes of the presented study, patients with significant cardiovascular disease diagnosed prior to hospitalization, such as at least grade 2 hypertension, heart failure, ischemic heart disease, or at least moderate valve disease, were included.

Renal function was expressed as the estimated glomerular filtration rate (eGFR). The eGFR was calculated by the abbreviated Modification of Diet in Renal Disease formula:

$$
\mathrm{eGFR}=186 \times \mathrm{SCr}-1.154 \times(\text { age })-0.203 \times(0.742 \text { iffemale })
$$

where, eGFR indicates estimated glomerular filtration rate $(\mathrm{mL} /$ $\mathrm{min} / 1.73 \mathrm{~m}^{2}$ ) and $\mathrm{SCr}$ is the serum creatinine level.

The following parameters were assessed by echocardiography: left-ventricular ejection fraction (LVEF), left-ventricular end-diastolic dimension (LVEDD), left-ventricular end-systolic dimension (LVESD), global longitudinal strain (GLS), and segmental contractility disorders. Echocardiography was performed in all patients within $48 \mathrm{~h}$ of admission using a General Electric Vingmed Echocardiography system with a $2.5-\mathrm{MHz}$ phased-array transducer. The LVEF was calculated using the universal formula: EF = (LVEDV - LVESV)/LVEDV $\times 100 \%$, where LVEDV indicates leftventricular end-diastolic volume and LVESV is the left-ventricular end-systolic volume. LVEDV and LVESV were calculated using 
Table 1. Detailed characteristics of the study group

\begin{tabular}{|c|c|c|c|c|c|c|c|}
\hline & Mean & Median & SD & Minimum & Maximum & Shapiro-Wilk & $95 \% \mathrm{Cl}$ \\
\hline MMP-2, ng/mL & 208 & 192 & 62.97 & 133 & 446 & $<0.00001$ & $190-225$ \\
\hline MMP-9, ng/mL & 851 & 799 & 438 & 166 & 1,946 & 0.0159 & $736-966$ \\
\hline $\mathrm{MMP}-3, \mathrm{ng} / \mathrm{mL}$ & 18.41 & 16.33 & 8.71 & 5.12 & 49.88 & 0.00061 & $16.06-20.76$ \\
\hline TIMP-2, ng/mL & 91.87 & 84.85 & 27.25 & 56.6 & 183 & 0.00006 & $84.64-99.10$ \\
\hline Age, years & 36.76 & 33 & 13.88 & 18 & 70 & 0.00021 & $33.11-40.41$ \\
\hline $\mathrm{CRP}, \mathrm{mg} / \mathrm{L}$ & 45.60 & 26.47 & 59.87 & 0.12 & 303 & $<0.00001$ & $29.71-61.49$ \\
\hline $\mathrm{BNP}, \mathrm{pg} / \mathrm{mL}$ & 254 & 55.1 & 529 & 10 & 2,197 & $<0.00001$ & $102-406$ \\
\hline Troponin, ng/mL & 9.09 & 4.97 & 11.63 & 0.01 & 44.8 & $<0.00001$ & $6.77-18.36$ \\
\hline Leukocytes, $10^{3} / \mu \mathrm{L}$ & 9.52 & 8.86 & 3.52 & 3.99 & 18.82 & 0.00081 & $8.59-10.44$ \\
\hline Erythrocytes, $10^{6} / \mu \mathrm{L}$ & 4.79 & 4.84 & 0.59 & 3.12 & 5.82 & 0.0376 & $4.64-4.95$ \\
\hline Hematocrit, $\%$ & 42.3 & 42.3 & 4.60 & 29.1 & 50 & 0.0259 & $41.09-43.51$ \\
\hline Hemoglobin, g/dL & 14.4 & 14.55 & 1.81 & 9.4 & 17.4 & 0.0964 & $13.92-14.87$ \\
\hline Potassium, $\mathrm{mmol} / \mathrm{L}$ & 4.21 & 4.19 & 0.46 & 3.1 & 5.35 & 0.816 & $4.09-4.33$ \\
\hline Sodium, $\mathrm{mmol} / \mathrm{L}$ & 139 & 139 & 2.41 & 132 & 143 & 0.0409 & $138-140$ \\
\hline Magnesium, mg/dL & 2.15 & 2.10 & 0.23 & 1.76 & 2.7 & 0.0992 & $2.09-2.21$ \\
\hline Creatinine, mg/dL & 0.97 & 0.96 & 0.17 & 0.68 & 1.45 & 0.0969 & $0.92-1.01$ \\
\hline eGFR & 89.64 & 92 & 23.85 & 6 & 132 & 0.0005 & $83.37-95.91$ \\
\hline LVEF, \% & 54.29 & 60 & 14.56 & 15 & 75 & 0.00109 & $50.47-58.12$ \\
\hline LVESD, mm & 36.5 & 35 & 8.99 & 22 & 62 & 0.00009 & $34.14-38.86$ \\
\hline LVEDD, mm & 53.64 & 52 & 6.53 & 42 & 70 & 0.00269 & $51.92-55.36$ \\
\hline GLS, \% & -15.52 & -16.5 & 5.56 & -24.3 & -1.1 & 0.0414 & -17.60 to $(-13.44)$ \\
\hline MR LVEF, \% & 55.85 & 59 & 13.47 & 12 & 75 & 0.00012 & $51.8-59.9$ \\
\hline MR LVESD, mm & 38.38 & 36 & 8.02 & 29 & 64 & 0.00003 & $3.60-4.08$ \\
\hline MR LVEDD, mm & 53.98 & 54 & 5.66 & 44 & 70 & 0.011 & $5.23-5.57$ \\
\hline
\end{tabular}

95\% Cl, 95\% confidence interval; BNP, B-type natriuretic peptide; CRP, C-reactive protein; eGFR, estimated glomerular filtration rate; GLS, echocardiographic global longitudinal strain; LVEDD, left-ventricular end-diastolic dimension in echocardiography; LVEF, left-ventricular ejection fraction in echocardiography; LVESD, left-ventricular end-systolic dimension in echocardiography; MMP, matrix metalloproteinase; MR LVEDD, left-ventricular end-diastolic dimension in cardiac magnetic resonance imaging; MR LVEF, left-ventricular ejection fraction in cardiac magnetic resonance imaging; MR LVESD, left-ventricular end-systolic dimension in cardiac magnetic resonance imaging; SD, standard deviation; Shapiro-Wilk, Shapiro-Wilk normality test; TIMP, tissue inhibitor of metalloproteinases.

the Simpson biplane method, in which the machine computes the left-ventricular volume based on perpendicular cross-sections of the left ventricle on the apical 2- and 4-chamber view, as determined by the operator.

Coronary angiography was performed in 29 patients, showing epicardial arterial changes in 2 of them. Cardiac MRI was also used to assess LVEF, LVEDD, LVESD, and myocarditis-specific features, including swelling and late gadolinium enhancement using a General Electric Signa HDxT 1.5T scanner. The final analysis included 45 patients ( 7 women and 38 men), comprising 11 with chronic kidney disease (CKD), who had cardiac MRI. Thirteen patients did not have an examination due to prior diagnosis, contraindications (presence of magnetic material), disagreement, or lack of co-operation of the patient. Among patients undergoing cardiac MRI, 3 did not have contrast during the study, and late gadolinium enhancement was confirmed in the subepicardial layer indicating myocarditis in 25 patients. In addition, cardiomyopathy was diagnosed in 3 cases: 1 case each of ischemic, hypertrophic, and dilated cardiomyopathy. The detailed characteristics of the studied group are presented in Table 1.

MMP-2, MMP-3, MMP-9, and TIMP-2 in

Myocarditis

\section{Statistical Methods}

The analyzed variables were both of nominal nature, including dichotomous and interval variables. Basic descriptive statistics were determined for interval variables: mean, standard deviation, minimum and maximum values, and a $95 \%$ confidence interval.

Contingency tables were the basis of the statistical analysis of variables in nominal scales and Pearson's linear correlation matrices for interval variables. The statistical significance of the determined correlation coefficients $r$ was verified by the $t$ test, with the assumed confidence level $\alpha=0.05$. First, the general relationships between the analyzed variables were assessed using principal component analysis (PCA) [20]. The PCA model was estimated using the NIPALS iterative algorithm. The convergence criterion was set at 0.00001 , setting the maximum number of iterations equal to 50 . The number of components was defined by determining the maximum predictive relevance by $\mathrm{V}$-fold cross-validation, setting their maximum number at $V_{\max }=7$.

The resulting optimal PCA model was finally reduced to 2 components. The results of the PCA analysis are presented in Tables 2 and 3 . The loading plot $\mathrm{p} 1$ versus $\mathrm{p} 2$ allowed us to predetermine 
Table 2. Validity of variables based on the PCA

\begin{tabular}{|c|c|c|c|}
\hline Validity of variables & Class & Strength & Validity \\
\hline Significant cardiovascular disease YES & 1 & 0.675568 & 2 \\
\hline Significant cardiovascular disease NO & 2 & 0.675568 & 2 \\
\hline LVEF & & 0.611645 & 3 \\
\hline LVESD & & 0.598840 & 4 \\
\hline eGFR & & 0.571225 & 5 \\
\hline MMP-2 & & 0.571186 & 6 \\
\hline TIMP-2 & & 0.564665 & 7 \\
\hline ECHO contractility disorders YES & 1 & 0.480446 & 9 \\
\hline ECHO contractility disorders NO & 2 & 0.480446 & 9 \\
\hline LVEDD & & 0.476995 & 10 \\
\hline ECG Q wave NO & 1 & 0.452301 & 12 \\
\hline ECG Q wave YES & 2 & 0.452301 & 12 \\
\hline ECG ST depression NO & 1 & 0.443172 & 14 \\
\hline ECG ST depression YES & 2 & 0.443172 & 14 \\
\hline Age & & 0.409799 & 15 \\
\hline Male & 1 & 0.404991 & 17 \\
\hline Female & 2 & 0.404991 & 17 \\
\hline ECG ST elevation NO & 1 & 0.380145 & 19 \\
\hline ECG ST elevation YES & 2 & 0.380145 & 19 \\
\hline ECG inverted T wave YES & 1 & 0.299024 & 21 \\
\hline ECG inverted T wave NO & 2 & 0.299024 & 21 \\
\hline MMP-3 & & 0.258577 & 22 \\
\hline ECG BBB NO & 1 & 0.120286 & 24 \\
\hline ECG BBB YES & 2 & 0.120286 & 24 \\
\hline MMP-9 & & 0.103218 & 25 \\
\hline Creatinine & & 0.096657 & 26 \\
\hline
\end{tabular}

BBB, bundle branch block (right or left); eGFR, estimated glomerular filtration rate; LVEDD, left-ventricular end-diastolic dimension in echocardiography; LVEF, left-ventricular ejection fraction in echocardiography; LVESD, left-ventricular end-systolic dimension in echocardiography; MMP, matrix metalloproteinase; TIMP, tissue inhibitor of metalloproteinases; PCA, principal component analysis.

the variables that have the most significant impact on the built model and select the most significant correlations between them. The variables selected in this way were then subjected to further statistical evaluation. Based on the results of the PCA, the nominal dichotomous variable Significant cardiovascular disease (YES/ $\mathrm{NO}$ ) was considered the basic grouping variable. To determine its correlation with other variables, univariate logistic regression was used with the free term $\neq 0$. The parameters of the logit function were estimated by the method of least squares using the QuasiNewton algorithm at the initial default parameters values of 0.1 , setting the initial step size equal to 0.01 . The convergence criterion for the logistic regression was set at 0.00001 . To assess the degree of correlation of the independent variables with the dichotomous dependent variable, we used the logistic regression model to calculate the odds ratios and their $95 \%$ confidence intervals. The statistical significance of built logistic models was assessed by the $\chi^{2}$ test for degrees of freedom $\mathrm{df}=1$ and an assumed level of significance $(\alpha)$ of 0.05 . Statistical analyses were performed using the STATISTICA PL ${ }^{\circledR}$ version 13 software.
Table 3. Univariate logistic regression analysis for a significant history of cardiovascular disease

\begin{tabular}{lllr}
\hline Variable & OR & \multicolumn{1}{l}{$95 \% \mathrm{Cl}$} & $p$ value \\
\hline MMP-2 & 1.020 & $1.006-1.034$ & 0.004 \\
MMP-3 & 1.092 & $1.009-1.182$ & 0.025 \\
TIMP-2 & 1.048 & $1.017-1.081$ & 0.002 \\
Age & 1.120 & $1.053-1.192$ & $<0.001$ \\
eGFR & 1.087 & $1.029-1.149$ & 0.002 \\
LVEF & 1.089 & $1.034-1.147$ & $<0.001$ \\
LVESD & 1.176 & $1.070-1.293$ & $<0.001$ \\
LVEDD & 1.213 & $1.077-1.366$ & 0.001 \\
GLS & 1.505 & $1.091-2.076$ & 0.009 \\
MR LVEF & 1.093 & $1.022-1.169$ & 0.008 \\
MR LVESD & 4.573 & $1.519-13.766$ & 0.005 \\
MR LVEDD & 7.194 & $1.483-34.902$ & 0.012 \\
Female sex & 11.143 & $2.627-47.264$ & 0.001 \\
Segmental contractility disorders & 12.133 & $2.412-61.031$ & 0.002 \\
\hline
\end{tabular}

95\% Cl, 95\% confidence interval; eGFR, estimated glomerular filtration rate; GLS, echocardiographic global longitudinal strain; LVEDD, left-ventricular end-diastolic dimension in echocardiography; LVEF, left-ventricular ejection fraction in echocardiography; LVESD, left-ventricular end-systolic dimension in echocardiography; MMP, matrix metalloproteinase; MR LVEDD, left-ventricular end-diastolic dimension in cardiac magnetic resonance imaging; MR LVEF, left-ventricular ejection fraction in cardiac magnetic resonance imaging; MR LVESD, left-ventricular end-systolic dimension in cardiac magnetic resonance imaging; TIMP, tissue inhibitor of metalloproteinases; OR, odds ratio.

\section{Results}

Using PCA, we determined the most important parameter differentiating the study group is the history of significant cardiovascular disease (Table 2; Fig. 1, 2). Univariate logistic regression analysis for significant cardiovascular disease showed a significant increased odds ratio for numerous parameters (Table 3 ). Patients with chronic kidney damage had higher levels of MMP-2 and TIMP2 , although they were not significantly different from those with healthy kidneys in terms of $\mathrm{N}$-terminal pro B-type natriuretic peptide levels and LVEF.

\section{Correlations}

We observed negative correlations between eGFR and MMP-2, eGFR, and MMP-3 and eGFR and TIMP-2 (Table 4). We also found creatinine positively correlated with MMP-3 concentration (Table 4). In addition, the correlation between MMP-2 and TIMP-2 levels in patients with chronic kidney damage did not differ significantly from those without. In multivariate canonical 
Fig. 1. Principal component analysis. CL, control limit; WL, warning limit.

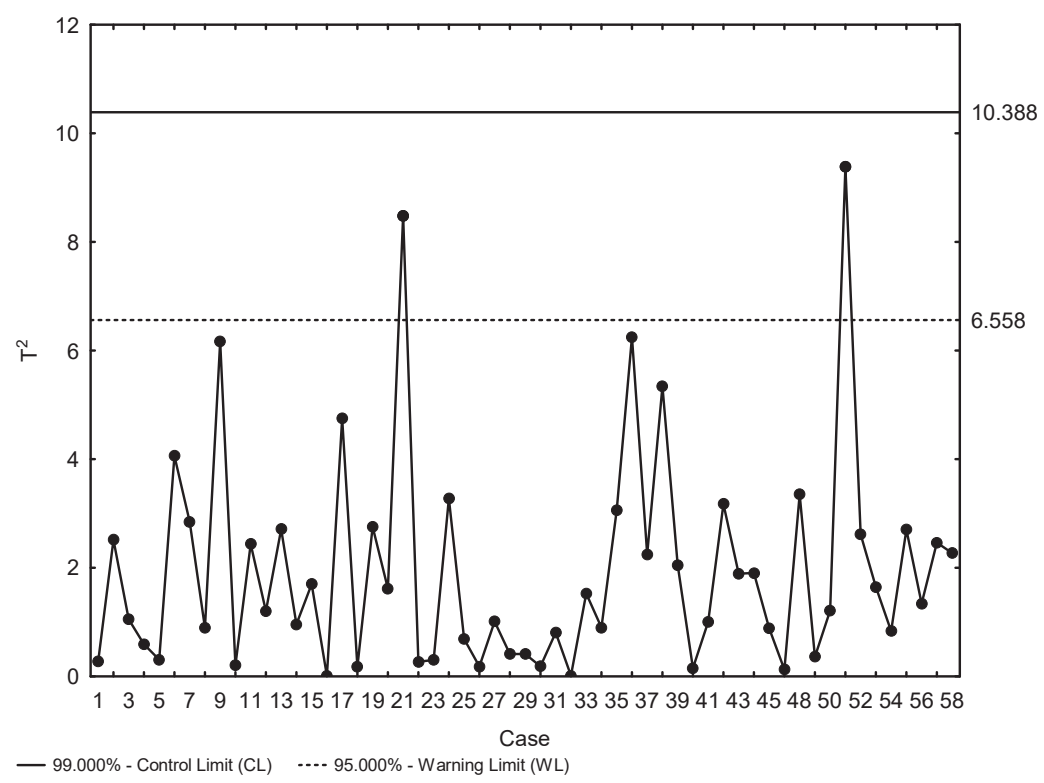

Table 4. Correlation of creatinine, eGFR, and MMP levels with the tissue inhibitor level

\begin{tabular}{ll}
\hline Creatinine & eGFR \\
\hline MMP-2 & \\
0.156 & $-\mathbf{0 . 5 3 5}$ \\
$p=0.269$ & $\boldsymbol{p}<\mathbf{0 . 0 0 1}$ \\
MMP-9 & -0.102 \\
0.083 & $p=0.470$ \\
$p=0.560$ & \\
MMP-3 & $-\mathbf{0 . 4 3 9}$ \\
0.464 & $\boldsymbol{p}=\mathbf{0 . 0 0 1}$ \\
$p=0.001$ & $-\mathbf{0 . 5 6 4}$ \\
TIMP-2 & $\boldsymbol{p}<\mathbf{0 . 0 0 1}$ \\
0.249 &
\end{tabular}

Bold values are statistically significant. eGFR, estimated glomerular filtration rate; MMP, matrix metalloproteinase; TIMP, tissue inhibitor of metalloproteinases.

analysis, we demonstrated a statistically significant correlation between the sets of variables: (1) MMP-2, MMP9, MMP-3, and TIMP-2 and (2) creatinine and eGFR (Table 5).

We found a statistically significant negative correlation between MMP-2, MMP-3, and TIMP-2 concentration with the LVEF (Table 6). Meanwhile, MMP-2, MMP-
Table 5. Multivariate canonical analysis of variables in group 1 (MMP-2, MMP-9, MMP-3, and TIMP-2) and in group 2 (creatinine and eGFR)

\begin{tabular}{lll}
\hline$N=52$ & \multicolumn{2}{l}{$\begin{array}{l}\text { Summary of canonical analysis } \\
R \text { canonical: } 0.63738\end{array}$} \\
& \multicolumn{2}{l}{$X^{2}(8)=34.888 p=0.00003$} \\
\cline { 2 - 3 } & left set & right set \\
\hline Variables, $n$ & 4 & 2 \\
Variance extracted, $\%$ & 69.2668 & 100.000 \\
Total redundancy, $\%$ & 24.2874 & 30.5627 \\
Variables & & \\
1 & MMP-2 $\mathrm{ng} / \mathrm{mL}$ & Creatinine $(\mathrm{mg} / \mathrm{dL})$ \\
2 & MMP-9 $\mathrm{ng} / \mathrm{mL}$ & eGFR \\
3 & MMP-3 $\mathrm{ng} / \mathrm{mL}$ & \\
4 & TIMP-2 $\mathrm{ng} / \mathrm{mg}$ &
\end{tabular}

eGFR, estimated glomerular filtration rate; MMP, matrix metalloproteinase.
3, and TIMP-2 levels positively correlated with LVESD. MMP-3 values also correlated positively with GLS. TIMP2 values correlated positively with LVEDD, GLS, and the LVESD assessed by MRI and correlated negatively with the LVEF assessed by MRI (Table 6). There were no significant correlations between MMP-2, MMP-3, MMP-9, and TIMP-2 with serum C-reactive protein. 


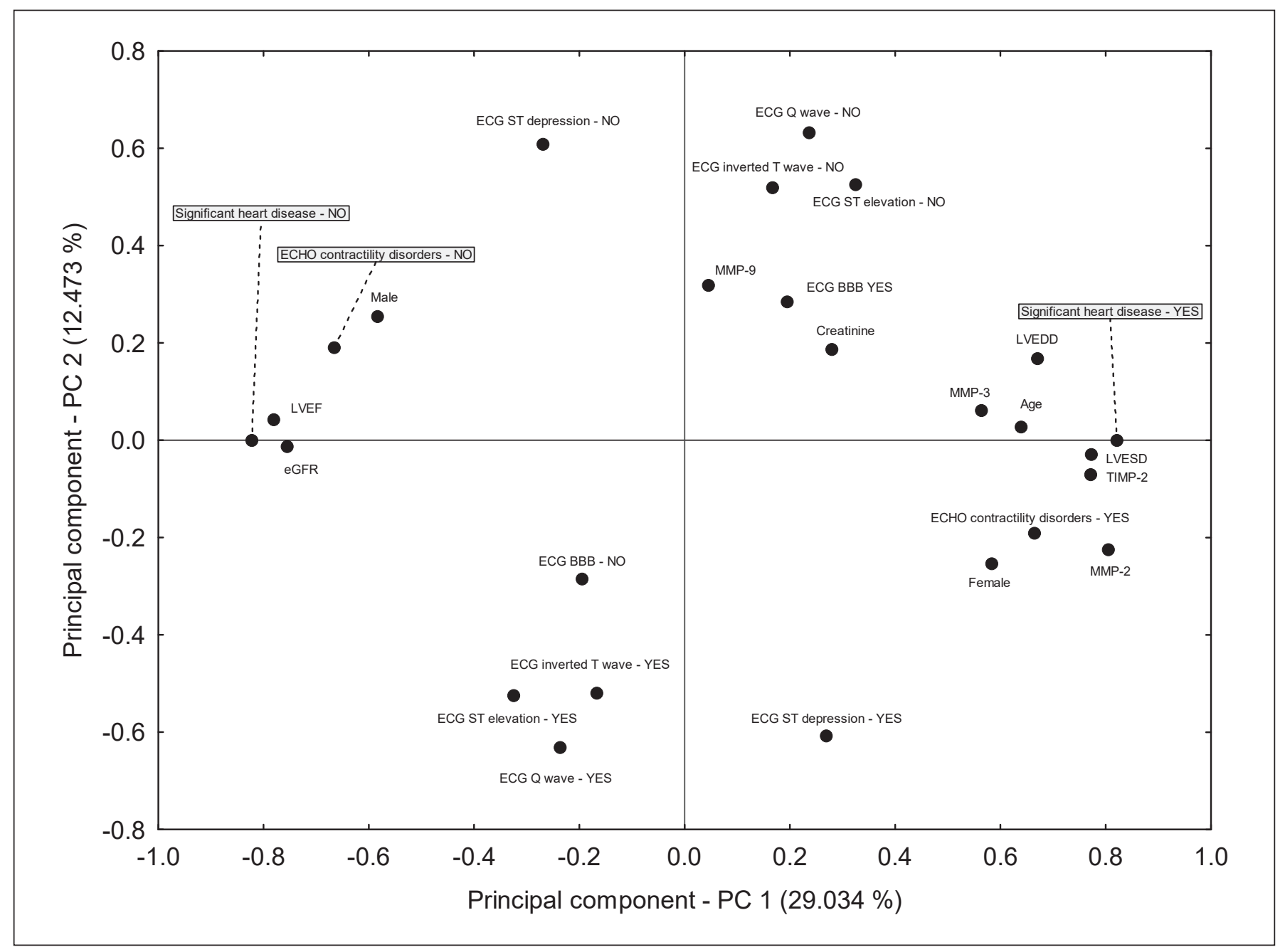

Fig. 2. Hotelling T2 multivariate control chart.

\section{Discussion}

Available data indicate extensive inflammatory processes exacerbate structural changes and may accelerate the development of heart failure [21-23]. In particular, increased collagen synthesis in the myocardium, along with its impaired degradation in response to pressure and volume overload, leads to collagen accumulation within the heart, causing structural remodeling, myocardial stiffness, and fibrosis [24, 25]. Indeed, biomarkers of myocardial inflammation and ECM remodeling, such as MMPs, have previously been shown to be important predictors of mortality [26].

Here, we found that serum MMP-2, MMP-3, and TIMP-2 levels were significantly negatively correlated with ejection fraction values in patients with myocarditis.
Elevated MMP-2 activity may lead to abnormal vascular remodeling by increasing the migration of vascular wall smooth muscle cells into the intima, increasing fibrosis and reducing the elastin content $[6,27]$. In addition, MMP-2 increases the activity, adhesion, and aggregation of platelets and thus contributes to thrombus formation [13]. Furthermore, in patients with myocardial infarction, a higher initial concentration of MMP-2 was associated with a larger infarct area and a reduced ejection fraction in an observation lasting over several months [28]. Therefore, our results showing elevated serum MMP-2 levels and reduced cardiac function, confirming the relationship already observed in patients with myocardial infarction.

We also observed a negative correlation between the eGFR and MMP-2 levels. A previous study showed high- 
Table 6. Correlation of biochemical values with the results of imaging studies in echocardiography and MRI

\begin{tabular}{|c|c|c|c|c|c|c|}
\hline \multicolumn{7}{|l|}{ MMP-2 } \\
\hline $\begin{array}{l}-0.555 \\
p=0.007\end{array}$ & $\begin{array}{l}0.539 \\
p=0.010\end{array}$ & $\begin{array}{l}0.229 \\
p=0.306\end{array}$ & $\begin{array}{l}0.407 \\
p=0.060\end{array}$ & $\begin{array}{l}-0.373 \\
p=0.087\end{array}$ & $\begin{array}{l}0.338 \\
p=0.124\end{array}$ & $\begin{array}{l}0.216 \\
p=0.334\end{array}$ \\
\hline \multicolumn{7}{|l|}{ MMP-9 } \\
\hline $\begin{array}{l}-0.218 \\
p=0.329\end{array}$ & $\begin{array}{l}0.308 \\
p=0.164\end{array}$ & $\begin{array}{l}0.196 \\
p=0.381\end{array}$ & $\begin{array}{l}0.253 \\
p=0.257\end{array}$ & $\begin{array}{l}-0.084 \\
p=0.711\end{array}$ & $\begin{array}{l}0.220 \\
p=0.325\end{array}$ & $\begin{array}{l}0.044 \\
p=0.845\end{array}$ \\
\hline $\begin{array}{l}-0.517 \\
p=0.014\end{array}$ & $\begin{array}{l}0.482 \\
p=0.023\end{array}$ & $\begin{array}{l}0.299 \\
p=0.177\end{array}$ & $\begin{array}{l}0.571 \\
p=0.006\end{array}$ & $\begin{array}{l}-0.319 \\
p=0.147\end{array}$ & $\begin{array}{l}0.324 \\
p=0.141\end{array}$ & $\begin{array}{l}0.284 \\
p=0.201\end{array}$ \\
\hline \multicolumn{7}{|l|}{ TIMP-2 } \\
\hline $\begin{array}{l}-0.685 \\
p<0.001\end{array}$ & $\begin{array}{l}0.668 \\
p=0.001\end{array}$ & $\begin{array}{l}0.426 \\
p=0.048\end{array}$ & $\begin{array}{l}0.540 \\
p=0.010\end{array}$ & $\begin{array}{l}-0.443 \\
p=0.039\end{array}$ & $\begin{array}{l}0.435 \\
p=0.043\end{array}$ & $\begin{array}{l}0.294 \\
p=0.184\end{array}$ \\
\hline
\end{tabular}

Bold values are statistically significant. GLS, echocardiographic global longitudinal strain; LVEDD, left-ventricular end-diastolic dimension in echocardiography; LVEF, left-ventricular ejection fraction in echocardiography; LVESD, left-ventricular end-systolic dimension in echocardiography; MMP, matrix metalloproteinase; MR LVEDD, left-ventricular end-diastolic dimension in cardiac magnetic resonance imaging; MR LVEF, left-ventricular ejection fraction in cardiac magnetic resonance imaging; MR LVESD, left-ventricular end-systolic dimension in cardiac magnetic resonance imaging; TIMP, tissue inhibitor of metalloproteinases; MRI, magnetic resonance imaging.

er MMP-2 levels were associated with episodes of cardiovascular disease in patients with type 1 diabetes observed for 12 years and that the baseline eGFR weakened the relationship between MMP-2 and cardiovascular disease [29]. Therefore, the eGFR may at least partially shape the relationship between MMPs and cardiovascular diseases. This hypothesis is supported by the observation that MMP-2 causes changes that are characteristic for tubular epithelial-to-mesenchymal transition of kidneys, leading to their increased fibrosis, which, in turn, leads to impaired renal function [30]. Alternatively, higher MMP-2 levels may result from reduced kidney function, although this is less likely as serum MMP-2 levels are only slightly dependent on renal clearance (the molecular weight of MMP-2 is higher than that of albumin).

In addition to the negative correlation between the eGFR and MMP-2, we observed a negative correlation between eGFR and MMP-3. MMP-3 concentration was also previously shown to be associated with a decrease in the eGFR; however, the relationship between MMP-3 and total mortality did not change significantly after adjustment to the decrease in the eGFR [31]. These results indicate increased concentrations of MMP-2 and -3 probably only partially contribute to the decrease in the eGFR, which correlates positively with cardiovascular disease and total mortality [31].

MMPs may be of particular concern in heart failure patients with concurrent CKD. Indeed, Du et al. [32] showed MMP-2 and MMP-9 levels increased over time in damaged tubules and lead to renal fibrosis, which is a typical phenomenon in progressive renal disease. Other studies have shown MMP-2 plays a pathological role in interstitial renal fibrosis, probably by inducing epithelial-to-mesenchymal transition and macrophage infiltration [33]. In addition, Chen et al. [34] showed increased MMP-2, MMP-9, and TIMP-1 activity in the aorta of patients with progressive kidney disease and increased activity of MMP-2 in the serum. Increased tissue activity of MMP-2, MMP-9, and TIMP-2 levels were also demonstrated in rats with CKD, with elevated TIMP-2 levels likely compensating for the increased MMP activity. There was also a strong relationship between MMP-3 levels and albuminuria in rats with CKD, presumably due to increased MMP-3-dependent proteolysis of type IV collagen [17]. Furthermore, parallel studies in patients with CKD showed increased MMP-2 activity in arterial blood samples of patients who underwent kidney transplantation accompanied by vascular calcification [30].

The Hsu et al. [35] study demonstrated that baseline MMP-2, -3, and -9 levels were independent predictors for faster eGFR decline and subsequent kidney disease progression. Their statistical analysis showed also that a low basal eGFR and higher MMP-9 levels were the independent predictors of mortality in CAD patients [35].

In our study, patients with chronic kidney damage had higher levels of MMP-2 and TIMP-2, although they were not significantly different from those with healthy kid- 
neys in terms of N-terminal pro B-type natriuretic peptide levels and LVEF. In addition, the correlation between MMP-2 and TIMP-2 levels in patients with chronic kidney damage did not differ significantly from those without, indicating that balance is maintained between the MMP and its inhibitor in those with kidney damage. Although such activation of MMP activity has already been observed in the cardiopulmonary setting and in severe inflammatory processes, our study is one of the first to show that chronic kidney damage in patients with myocarditis increases MMP activity. Therefore, inhibition of MMPs may be a useful therapeutic strategy in patients with myocarditis and concomitant CKD.

The future novel medical strategies regulating and controlling MMP activity may help to elucidate the precise ways for conquering kidney diseases. Further progress in mastering control and regulation of MMP actions depends on the development of certain MMP inhibitors and finding specific MMP-targeted therapeutic strategies [36].

\section{Limitations}

There are several limitations to our study. First, plasma levels of MMP-2, $-3,-9$, and of TIMP-2 were only determined at baseline. Therefore, the changes in plasma levels over time, revealing more details about outcome measures, are not known. Second, we do not know to what extent plasma levels reflect the local pathological situation at the tissue level. Finally, we only assessed a few MMPs and only one of the 4 known TIMPs, so other MMPs and TIMPs may be involved in this pathogenic process.

\section{Conclusion}

1. We demonstrated that serum MMP-2, MMP-3, and TIMP-2 concentrations were related to LVEF, and MMP-3 levels correlated with longitudinal deformation, indicating MMPs play an important role in the post-inflammatory remodeling of the myocardium.

2. A history of cardiovascular disease, including at least grade 2 hypertension, heart failure, ischemic heart disease, and at least moderate valve disease, was an important element in modifying the relationship between MMPs and the degree of myocardial damage.

3. A negative correlation between the eGFR and MMP-2, MMP-3, and TIMP-2 and a positive correlation between creatinine and MMP-3 levels indicate the role of MMPs and TIMP-2 in renal dysfunction.

\section{Acknowledgment}

The authors thank Proper Medical Writing Sp. z o.o. for language assistance provided in the preparation of this paper.

\section{Statement of Ethics}

This study was conducted in accordance with the Helsinki Declaration. The study design was accepted by the Bioethics Committee of the Wroclaw Medical University. Written informed consent was obtained from each individual subject included in the study. The study approval reference number KB-107/2016.

\section{Conflict of Interest Statement}

The authors have no conflicts of interest that are relevant to the content of this article. The authors have no relevant financial or nonfinancial interests to disclose.

\section{Funding Sources}

Uniwersytet Medyczny im. Piastów Slaskich we Wroclawiu Grant ST-908/SUB.C150.15.068.

\section{Author Contributions}

All the authors contributed to the study conception and design. Material preparation, data collection, and analysis were performed by Małgorzata Kobusiak-Prokopowicz, Konrad Kaaz, Dominik Marciniak, Bożena Karolko, and Andrzej Mysiak. The first draft of the manuscript was written by Małgorzata Kobusiak-Prokopowicz and Konrad Kaaz, and all the authors commented on previous versions of the manuscript. All the authors read and approved the final manuscript.

\section{Data Availability Statement}

The data that support the findings of this study are available from the corresponding author, upon reasonable request.

\section{References}

Kidney Blood Press Res 2021;46:749-757 DOI: $10.1159 / 000519594$
1 Graham HK, Horn M, Trafford AW. Extracellular matrix profiles in the progression to heart failure. European young physiologists symposium keynote lecture-Bratislava 2007. Acta Physiol. 2008 Sep;194(1):3-21.

2 Wilson EM, Spinale FG. Myocardial remodelling and matrix metalloproteinases in heart failure: turmoil within the interstitium. Ann Med. 2001 Dec;33(9):623-34.

3 Woessner JF Jr. Matrix metalloproteinases and their inhibitors in connective tissue remodeling. FASEB J. 1991 May;5(8):2145-54.

Kobusiak-Prokopowicz/Kaaz/Marciniak/ Karolko/Mysiak 
4 MacColl E, Khalil RA. Matrix metalloproteinases as regulators of vein structure and function: implications in chronic venous disease. J Pharmacol Exp Ther. 2015 Dec;355(3):41028.

5 Fingleton B, Matrisian LM. Matrix metalloproteinases as targets for therapy in Kaposi sarcoma. Curr Opin Oncol. 2007;13(3):36873.

6 Chen Q, Jin M, Yang F, Zhu J, Xiao Q, Zhang L. Matrix metalloproteinases: inflammatory regulators of cell behaviors in vascular formation and remodeling. Mediators Inflamm. 2013;2013:928315.

7 Schulze CJ, Wang W, Suarez-Pinzon WL, Sawicka J, Sawicki G, Schulz R. Imbalance between tissue inhibitor of metalloproteinase-4 and matrix metalloproteinases during acute myocardial [correction of myoctardial] ischemia-reperfusion injury. Circulation. 2003 May 20;107(19):2487-92.

8 Hendry RG, Bilawchuk LM, Marchant DJ. Targeting matrix metalloproteinase activity and expression for the treatment of viral myocarditis. J Cardiovasc Transl Res. 2014 Mar; $7(2): 212-25$.

9 Westermann D, Lindner D, Kasner M, Zietsch C, Savvatis K, Escher F, et al. Cardiac inflammation contributes to changes in the extracellular matrix in patients with heart failure and normal ejection fraction. Circ Heart Fail. 2011 Jan;4(1):44-52.

10 Creemers EE, Cleutjens JP, Smits JF, Daemen MJ. Matrix metalloproteinase inhibition after myocardial infarction: a new approach to prevent heart failure? Circ Res. 2001 Aug 3;89(3): 201-10.

11 Jefferis BJ, Whincup P, Welsh P, Wannamethee G, Rumley A, Lennon L, et al. Prospective study of matrix metalloproteinase- 9 and risk of myocardial infarction and stroke in older men and women. Atherosclerosis. 2010 Feb;208(2):557-63.

12 Spinale FG. Matrix metalloproteinases: regulation and dysregulation in the failing heart. Circ Res. 2002 Mar 22;90(5):520-30.

13 Gresele P, Falcinelli E, Loffredo F, Cimmino G, Corazzi T, Forte L, et al. Platelets release matrix metalloproteinase- 2 in the coronary circulation of patients with acute coronary syndromes: possible role in sustained platelet activation. Eur Heart J. 2011 Feb;32(3):31625.

14 Chase AJ, Newby AC. Regulation of matrix metalloproteinase (matrixin) genes in blood vessels: a multi-step recruitment model for pathological remodelling. J Vasc Res. 2003 Jul-Aug;40(4):329-43.

15 Giebel SJ, Menicucci G, McGuire PG, Das A. Matrix metalloproteinases in early diabetic retinopathy and their role in alteration of the blood-retinal barrier. Lab Invest. 2005 May; 85(5):597-607.
16 Ebihara I, Nakamura T, Shimada N, Koide H. Increased plasma metalloproteinase- 9 concentrations precede development of microalbuminuria in non-insulin-dependent diabetes mellitus. Am J Kidney Dis. 1998 Oct;32(4): 544-50.

17 Hosoyamada Y, Sakai T. Structural arrangement of collagen fibrils in the periarterial connective tissue of the kidney: their functional relevance as a structural stabilizer against arterial pressure. Anat Sci Int. 2012 Jun;87(2): 80-7.

18 Cheng S, Lovett DH, Gelatinase A (MMP-2) is necessary and sufficient for renal tubular cell epithelial-mesenchymal transformation. Am J Pathol. 2003 Jun;162(6):1937-49.

19 Caforio AL, Pankuweit S, Arbustini E, Basso C, Gimeno-Blanes J, Felix SB, et al.; European Society of Cardiology Working Group on Myocardial and Pericardial Diseases. Current state of knowledge on aetiology, diagnosis, management, and therapy of myocarditis: a position statement of the European Society of Cardiology Working Group on Myocardial and Pericardial Diseases. Eur Heart J. 2013 Sep;34(33):2636-48, 2648a-2648d.

20 Zhang Z, Castelló A. Principal components analysis in clinical studies. Ann Transl Med. 2017 Sep;5(17):351.

21 Boulogne M, Sadoune M, Launay JM, Baudet M, Cohen-Solal A, Logeart D. Inflammation versus mechanical stretch biomarkers over time in acutely decompensated heart failure with reduced ejection fraction. Int J Cardiol. 2017 Jan 1;226:53-9.

22 AbouEzzeddine OF, McKie PM, Scott CG Rodeheffer RJ, Chen HH, Michael Felker G, et al. Biomarker-based risk prediction in the community. Eur J Heart Fail. 2016 Nov; 18(11):1342-50.

23 Jackson CE, Haig C, Welsh P, Dalzell JR, Tsorlalis IK, McConnachie A, et al. The incremental prognostic and clinical value of multiple novel biomarkers in heart failure. Eur J Heart Fail. 2016 Dec;18(12):1491-8.

24 Morishita T, Uzui H, Mitsuke Y, Amaya N, Kaseno K, Ishida K, et al. Association between matrix metalloproteinase-9 and worsening heart failure events in patients with chronic heart failure. ESC Heart Fail. 2017 Aug;4(3): 321-30.

25 Täger T, Wiebalck C, Fröhlich H, Corletto A, Katus HA, Frankenstein L. Biological variation of extracellular matrix biomarkers in patients with stable chronic heart failure. Clin Res Cardiol. 2017;106:974-85.

26 Dörr O, Walther C, Liebetrau C, Keller T, Tabert H, Boeder N, et al. Specific biomarkers of myocardial inflammation and remodeling processes as predictors of mortality in highrisk patients undergoing percutaneous mitral valve repair (MitraClip). Clin Cardiol. 2018 Apr;41(4):481-7.
27 Lan TH, Huang XQ, Tan HM. Vascular fibrosis in atherosclerosis. Cardiovasc Pathol. 2013 Sep-Oct;22(5):401-7.

28 Nilsson L, Hallén J, Atar D, Jonasson L, Swahn E. Early measurements of plasma matrix metalloproteinase-2 predict infarct size and ventricular dysfunction in ST-elevation myocardial infarction. Heart. 2012 Jan;98(1): 31-6.

29 Peeters SA, Engelen L, Buijs J, Jorsal A, Parving HH, Tarnow L, et al. Plasma matrix metalloproteinases are associated with incident cardiovascular disease and all-cause mortality in patients with type 1 diabetes: a 12-year follow-up study. Cardiovasc Diabetol. 2017 Apr 26;16(1):55.

30 Chung AW, Yang HH, Kim JM, Sigrist MK, Chum E, Gourlay WA, et al. Upregulation of matrix metalloproteinase-2 in the arterial vasculature contributes to stiffening and vasomotor dysfunction in patients with chronic kidney disease. Circulation. 2009;120(9):792801.

31 Peeters SA, Engelen L, Buijs J, Chaturvedi N, Fuller JH, Schalkwijk CG, et al.; EURODIAB Prospective Complications Study Group. Plasma levels of matrix metalloproteinase-2, $-3,-10$, and tissue inhibitor of metalloproteinase- 1 are associated with vascular complications in patients with type 1 diabetes: the EURODIAB Prospective Complications Study. Cardiovasc Diabetol. 2015 Mar 10;14:31.

32 Du X, Shimizu A, Masuda Y, Kuwahara N, Arai T, Kataoka M, et al. Involvement of matrix metalloproteinase- 2 in the development of renal interstitial fibrosis in mouse obstructive nephropathy. Lab Invest. 2012 Aug;92(8): 1149-60.

33 Yu XA, Hu Y, Zhang Y, Zhang R, Bai X, Gu L, et al. Integrating the polydopamine nanosphere/aptamers nanoplatform with a $\mathrm{DN}$ ase-I-assisted recycling amplification strategy for simultaneous detection of MMP-9 and MMP-2 during renal interstitial fibrosis. ACS Sens. 2020 Apr 24;5(4):1119-25.

34 Chen NX, O'Neill KD, Chen X, Kiattisunthorn K, Gattone VH, Moe SM. Activation of arterial matrix metalloproteinases leads to vascular calcification in chronic kidney disease. Am J Nephrol. 2011;34(3):211-9.

35 Hsu TW, Kuo KL, Hung SC, Huang PH, Chen JW, Tarng DC. Progression of kidney disease in non-diabetic patients with coronary artery disease: predictive role of circulating matrix metalloproteinase-2, -3, and -9. PLoS One. 2013;8(7):e70132.

36 Zakiyanov O, Kalousová M, Zima T, Tesař V. Matrix metalloproteinases in renal diseases: a critical appraisal. Kidney Blood Press Res. 2019;44(3):298-330. 